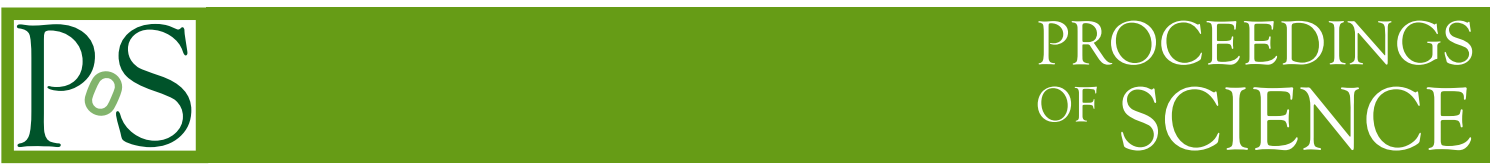

\title{
Neutrinoless double beta decay: Theory challenges
}

\author{
Fedor Šimkovic* \\ Department of Nuclear Physics and Biophysics, Comenius University, Bratislava, Slovakia \\ Laboratory of Theoretical Physics, JINR, 141980 Dubna, Moscow region, Russia \\ Czech Technical University in Prague, 128-00 Prague, Czech Republic \\ E-mail: simkovicefmph.uniba.sk
}

\begin{abstract}
The recent progress in theoretical description of the neutrinoless double beta decay ( $0 v \beta \beta$-decay) is briefly reviewed. By taking advantage of an interpolating formula, which allows to calculate the $0 v \beta \beta$-decay half-life for an arbitrary neutrino mass and which is expressed with a single nuclear matrix element, neutrino mass mechanisms of this process having origin in the left-right symmetric models are discussed. To understand the importance of the mass mechanism due to right-handed leptonic current, an assumption about seesaw-type $6 \times 6$ unitary neutrino mixing matrix and relation of light and heavy neutrino masses is made. The region of dominance of right over left leptonic current mechanisms is identified. Further, a progress in the calculation of double beta decay nuclear matrix elements within the quasiparticle random phase approximation is reported. A connection between the two neutrino double-beta decay and $0 v \beta \beta$-decay matrix elements is discussed. An impact of the quenching of the axial-vector coupling constant $g_{A}$ on double-beta decay processes is addressed and a possibility to determine quenched value of $g_{A}$ is announced.
\end{abstract}

Neutrino Oscillation Workshop (NOW2018)

9 - 16 September, 2018

Rosa Marina (Ostuni, Brindisi, Italy)

${ }^{*}$ Speaker. 


\section{Introduction}

With the discovery of Higgs boson at Large Hadron Collider the Standard Model (SM) of particle physics is complete. At the same time many theoretical questions including those about the origin of neutrino masses remain unanswered. Small masses of neutrinos observed in neutrino oscillations experiments imply that SM, which is a spontaneously broken gauge theory, needs be extrapolated well above electroweak scale. Recall that the SM neutrinos are strictly massless.

As neutrinos are the only electrically neutral SM fermions the neutrino mass generation can be different from the standard Higgs mechanism. A popular scenario is that neutrinos are light as a result of their Majorana nature. A subject of interest is a unique dimension $d=5$ Weinberg operator, namely a non-renormalizable interaction, that produces Majorana neutrino masses and generally implies lepton number violation. The see-saw mechanism, which induces light neutrino masses from heavy neutral fermion exchange, is the most popular way to resolve the origin of those terms.

The most sensitive experiments to the Majorana masses and nature of neutrinos are those searching for a signal of the neutrinoless double beta decay ( $0 v \beta \beta$-decay),

$$
(A, Z) \rightarrow(A, Z+2)+2 e^{-} .
$$

No signal has been observed so far, but impressive half-life limits of more than $10^{25-26}$ years have been achieved in several experiments on different target nuclei. Search for the $0 v \beta \beta$ decay is at the forefront of the present day nuclear and particle physics.

If masses of neutral heavy fermions are at the GUT scale, the light-neutrino exchange produced by left-handed currents is the driving mechanism for $0 v \beta \beta$-decay. The inverse half-life of the $0 v \beta \beta$-decay is given by [1]

$$
\left[T_{1 / 2}^{0 v}\right]^{-1}=G^{0 v}\left(g_{A}^{\text {eff }}\right)^{4}\left|M_{v}^{0 v}\right|^{2} \frac{\left|m_{\beta \beta}\right|^{2}}{m_{e}^{2}}
$$

where $G^{0 v}, g_{A}^{\text {eff }}$ and $M^{0 v}$ represent the known phase-space factor, the effective axial-vector coupling constant and the nuclear matrix element (NME) of the process, respectively. The ultimate goal of the search for $0 v \beta \beta$-decay is to determine the Majorana neutrino mass,

$$
m_{\beta \beta}=\left|\sum_{j=1}^{3} U_{e j}^{2} m_{j}\right|,
$$

Here, $U_{e j}$ and $m_{j}(j=1,2,3)$ are elements of Pontecorvo-Maki-Nakagawa-Sakata (PMNS) neutrino mixing matrix and masses of neutrinos, respectively. To infer the value of lepton number violating parameter $(\mathrm{LNV}) m_{\beta \beta}$ from a non-zero $0 v \beta \beta$-decay rate measurement, $M^{0 v}$ and $g_{A}^{\text {eff }}$ have to be reliably calculated by the tools of nuclear structure theory.

It is not known at what energy scale the new physics behind non-zero neutrino mass exists. Currently, models that connect neutrino physics to the TeV scale are widely discussed in the literature. As an example one can have left-right symmetric (LRS) theories, which lead to processes with lepton number violation at this energy scale and offer additional contributions to the $0 v \beta \beta$-decay rate due right-handed currents and heavy neutrinos. 
The focus of this contribution is on theoretical challenges of the $0 v \beta \beta$-decay as follows: i) Is there a dominance of mass mechanism due to right-handed leptonic current in the decay rate? ii) What needs to be understood for a reliable calculation of the $0 v \beta \beta$-decay NMEs? iii) What is the value of $g_{A}^{\text {eff? }}$ ?

\section{Interpolating formula for the $0 v \beta \beta$-decay half-life}

The LRS models extend the SM gauge symmetry to the group $S U(2)_{L} \otimes S U(2)_{R} \otimes U(1)_{B-L}$ with an additional neutral $Z^{\prime}$ and two charged vector bosons $W_{R}^{ \pm}$. This symmetry group is broken spontaneously down to the SM group at a sufficiently high energy scale $\Lambda_{L R}$ providing to the extra gauge bosons $Z^{\prime}, W_{R}^{ \pm}$large masses $\sim \Lambda_{L R}$. After integrating out these heavy particles one ends up at low energies with the well known current-current effective Lagrangian involving both left- and right-handed weak currents. The part, which can trigger the $0 v \beta \beta$-decay is

$$
\mathscr{L}^{\beta}=\frac{G_{\beta}}{\sqrt{2}}\left[j_{L}^{\rho} J_{L \rho}^{\dagger}+\lambda j_{R}^{\rho} J_{R \rho}^{\dagger}+\text { h.c. }\right] .
$$

Here, $G_{\beta}=G_{F} \cos \theta_{C}$, where $G_{F}$ and $\theta_{C}$ are Fermi constant and Cabbibo angle, respectively. $\lambda=\left(M_{W_{L}} / M_{W_{R}}\right)^{2}$, where $M_{W_{L}}$ and $M_{W_{R}}\left(M_{W_{L}}<M_{W_{R}}\right)$ are masses of $W_{L}$ and $W_{R}$ gauge bosons, respectively. The explicit form of the left/right-handed hadronic current $J_{L, R}^{\dagger}$ in nuclei can be found in [2]. The left/right-handed leptonic $j_{L / R}$ current is given by

$$
j_{L}^{\rho}=\bar{e}_{L} \gamma^{\rho}\left(\sum_{j=1}^{3} U_{e j} v_{j L}+\sum_{k=1}^{n} S_{e k}\left(N_{k L}\right)^{C}\right), j_{R}^{\rho}=\bar{e}_{R} \gamma^{\rho}\left(\sum_{j=1}^{3} T_{e j}^{*}\left(v_{j L}\right)^{C}+\sum_{k=1}^{n} V_{e k}^{*} N_{k R}\right) .
$$

The $3 \times 3$ block matrices in flavor space $U, S, T, V$ form a $6 \times 6$ unitary neutrino mixing matrix

$$
\mathscr{U}=\left(\begin{array}{cc}
U & S \\
T & V
\end{array}\right)
$$

which is a generalization of the PMNS matrix. $v_{j}$ and $N_{k}$ are the light and heavy Majorana mass eigenstate neutrinos with masses $m_{j}$ and $M_{j}$, respectively.

We assume the dominance of the neutrino mass mechanisms in $0 v \beta \beta$-decay originating from the mass term in the neutrino propagator connecting two vertices both with $W_{L}$ (standard mechanism) as well as both with $W_{R}$ ( $\lambda^{2}$ mechanism). This is motivated by the recent analysis [3] of the mixed $W_{L}-W_{R}$-contribution ( $\lambda$ mechanism) via the momentum dependent part of the neutrino propagator, which indicates its subdominant role in $0 v \beta \beta$-decay. For sake of simplicity the possible $W_{L}-W_{R}$-mixing is not taken into account.

Recently, it was found that the ratio of nuclear matrix elements $M_{\mathrm{N}}^{0 v}$ and $M_{v}^{\prime 0 v}$ associated with light $\left(m_{i} \ll p_{\mathrm{F}}\right)$ and heavy $\left(M_{i} \gg p_{\mathrm{F}}\right)$ neutrino mass mechanisms, respectively, weakly depends on the isotope. The mean square momentum of the virtual neutrino propagating between two $\beta$ decaying nucleons was introduced as follows:

$$
\left\langle p^{2}\right\rangle=m_{\mathrm{p}} m_{\mathrm{e}} \frac{M_{\mathrm{N}}^{\prime 0 v}}{M_{v}^{\prime 0 v}} .
$$


Here, $m_{e}\left(m_{p}\right)$ is the mass of electron (proton). The average value of mean square momentum $\left\langle p^{2}\right\rangle_{a}$ with variance $\sigma$ is given in [4].

For the $0 v \beta \beta$-decay half-life the "interpolating formula", which interpolates two limiting cases of light and heavy neutrinos and is valid to a good accuracy for an arbitrary value of neutrino mass $m_{j}$ and $M_{j}$, can be written as

$$
\left[T_{1 / 2}^{0 v}\right]^{-1}=\eta_{v N}^{2}\left(g_{\mathrm{A}}^{\mathrm{eff}}\right)^{4}\left|M_{v}^{0 v}\right|^{2} G^{0 v}=\frac{1}{m_{e}^{2}}\left(m_{\beta \beta}^{2}+\left(M_{\beta \beta}^{R}\right)^{2}\right)\left(g_{\mathrm{A}}^{\text {eff }}\right)^{4}\left|M_{v}^{0 v}\right|^{2} G^{0 v},
$$

where

$$
m_{\beta \beta}=\left|\sum_{j}^{3} U_{e j}^{2} m_{j}+\sum_{k}^{3} S_{e k}^{2} \frac{\left\langle p^{2}\right\rangle_{a}}{\left\langle p^{2}\right\rangle_{a}+M_{k}^{2}} M_{k}\right|, \quad M_{\beta \beta}^{R}=\lambda\left|\sum_{j}^{3} T_{e j}^{2} m_{j}+\sum_{k}^{3} V_{e k}^{2} \frac{\left\langle p^{2}\right\rangle_{a}}{\left\langle p^{2}\right\rangle_{a}+M_{k}^{2}} M_{k}\right| .
$$

In the limit case right-handed leptonic current is switched off $(\lambda=0), m_{\beta \beta}$ takes the form given in Eq. (1.3) and $M_{\beta \beta}^{R}=0$.

Within the LRS models the general LNV parameter $\eta_{v N}$ for light and heavy neutrino mass mechanisms can not be evaluated without additional assumptions. Recall that the $6 \times 6$ mixing matrix $\mathscr{U}$ in Eq. (2.3) is completely parameterized with 15 angles, 10 Dirac and 5 Majorana CP violating phases. If for a sake of simplicity the flavor universal mixing between the active and sterile neutrino sectors is considered, the seesaw type unitary mixing matrix $\mathscr{U}$ takes the form [4]

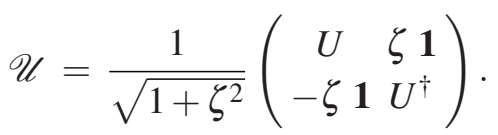

Here, $U$ is the PMNS unitary mixing matrix for light neutrinos. We note that mixing for heavy neutrinos is dictated to be hermitian conjugate of the PMNS matrix due to the unitary condition $\mathscr{U} \mathscr{U}^{\dagger}=\mathscr{U}^{\dagger} \mathscr{U}=\mathbf{1}$. Thus, the $6 \times 6$ unitary mixing matrix $\mathscr{U}$ in Eq. (2.7) is parametrized in terms of 3 mixing angles $\theta_{12}, \theta_{13}, \theta_{23}, 2$ Majorana CP phases $\alpha_{1}, \alpha_{2}, 1$ Dirac CP phase $\delta$ and the seessaw parameter $\zeta$, i.e., with a substantially less number of parameters as in the most general case.

In order to conclude about possible importance of $m_{\beta \beta}$ and $M_{\beta \beta}^{R}$ mechanisms, for neutrino masses let assume see-saw mass relation $m_{i} \simeq \zeta^{2} M_{i}\left(m_{i} \neq 0\right.$ and $\left.\zeta \ll 1\right)$. We obtain

$$
m_{\beta \beta}=\left|\sum_{j}^{3}\left(U_{e j}^{2}+\frac{\zeta^{4}\left\langle p^{2}\right\rangle_{a}}{\zeta^{4}\left\langle p^{2}\right\rangle_{a}+m_{j}^{2}}\right) m_{j}\right|, \quad M_{\beta \beta}^{R}=\frac{\lambda}{\zeta^{2}}\left|\sum_{j}^{3}\left(\zeta^{4}+\left(U_{e j}^{\dagger}\right)^{2} \frac{\zeta^{4}\left\langle p^{2}\right\rangle_{a}}{\zeta^{4}\left\langle p^{2}\right\rangle_{a}+m_{j}^{2}}\right) m_{j}\right| .
$$

We note that $m_{\beta \beta}\left(M_{\beta \beta}^{R}\right)$ depends on $\theta_{12}, \theta_{13}, \alpha_{1}, \alpha_{2}\left(\theta_{12}, \theta_{13}, \theta_{23}, \delta\right), m_{1,2,3}$ and $\zeta$. These quantities can be evaluated by making assumption about normal spectrum $\left(m_{1}<m_{2}<m_{3}\right)$ or inverted spectrum $\left(m_{3}<m_{1}<m_{2}\right)$ of neutrino masses and considering CP violating phases to be arbitrary. The current constrain on the mass of $W_{R}$ is $M_{W_{R}} \geq 2.9 \mathrm{TeV}$ [2] sets the limit $\lambda \leq 7.7 \times 10^{-4}$. The upper limit $\lambda=7.7 \times 10^{-4}$ will be used as a reference value for this parameter.

In Fig. 1 the region of dominance of $M_{\beta \beta}^{R}$ mechanism over $m_{\beta \beta}$ mechanism in the $0 v \beta \beta$-decay rate is displayed in the plane of parameters $\zeta$ and $m_{0}$ (the lightest neutrino mass). The cases of the normal (left panel) and inverted (right panel) mass spectrum of neutrino masses are presented. We 


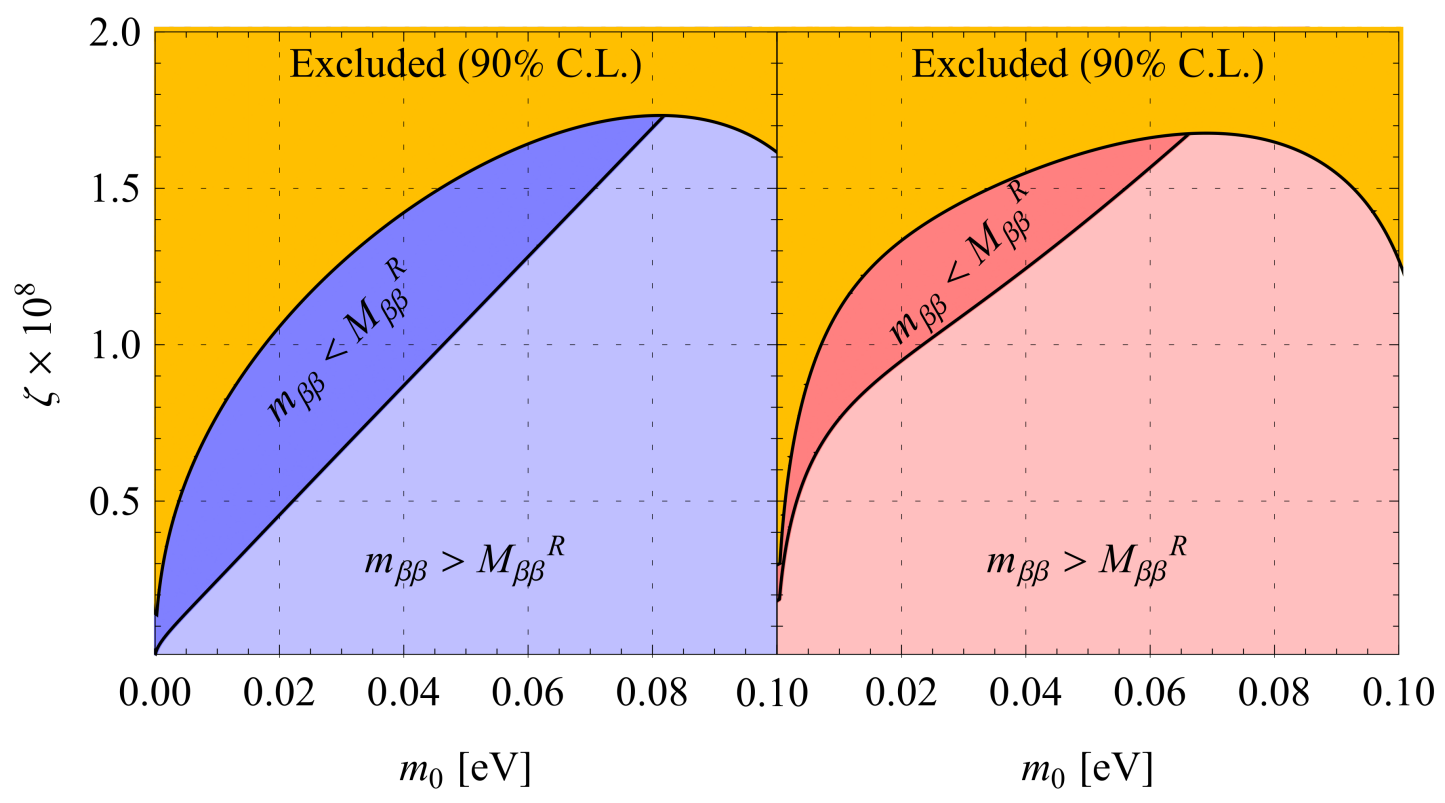

Figure 1: (Color online) The region of the dominance of the $M_{\beta \beta}^{R}$ contribution over $m_{\beta \beta}$ contribution to the LNV parameter $\eta_{v N}$ for the see-saw type of neutrino mixing matrix given in Eq. (2.7) and by assuming $\zeta^{2}=m_{i} / M_{i}$. The cases of the normal and inverted mass spectrum are presented in the left and right panels, respectively. The current constraint on the $\eta_{v N}$ parameter deduced from the lower limit on the $0 v \beta \beta$-decay half-life of ${ }^{136} \mathrm{Xe}$ with help of QRPA NME is considered [4].

see that for $m_{0} \gtrsim 0.8 \mathrm{eV} m_{\beta \beta}$ mechanism is dominant over $M_{\beta \beta}^{R}$ mechanism. For a lower value of $m_{0}$ there is a region governed by the $M_{\beta \beta}^{R}$ mechanism in spite of its significant suppression due to smallness of $\lambda$.

\section{Nuclear matrix element and effective axial-vector coupling constant}

In order to relate the observed $0 v \beta \beta$-decay half-life to $\eta_{v N}$, it is required to calculate reliably the nuclear matrix element $M_{v}^{0 v}$ within nuclear structure theory. This is a longstanding problem which has been discussed in a plethora of papers. The present status of calculation of $M_{v}^{0 v}$ is summarized in recent review [1].

The understanding of the two-neutrino double-beta decay $(A, Z) \rightarrow(A, Z+2)+2 e^{-}+2 \bar{v}_{e}$ ( $2 v \beta \beta$-decay) is crucial for a correct evaluation of $M_{v}^{0 v}$. In [5] it was showed that there is a close relation between the Gamow-Teller (GT) part of $M_{v}^{0 v}$ and the matrix element $M_{c l}^{2 v}$ of the experimentally observed $2 v \beta \beta$ decay, which is evaluated in the closure approximation. The importance of dependence of $M_{v}^{0 v}$ and $M_{\mathrm{cl}}^{2 v}$ on the distance $r$ between the two neutrons that are transformed in two protons in the double-beta decay was established. It was manifested that if this function, $C_{\mathrm{cl}}^{2 v}(r)$, is known for any particular mechanism of the $0 v \beta \beta$-decay, evaluation of the matrix element for any other mechanism is reduced to an integral over $\mathrm{r}$ of the product of two-nucleon exchange potential associated with a given LNV mechanism and $C_{\mathrm{cl}}^{2 v}(r)[5]$. 


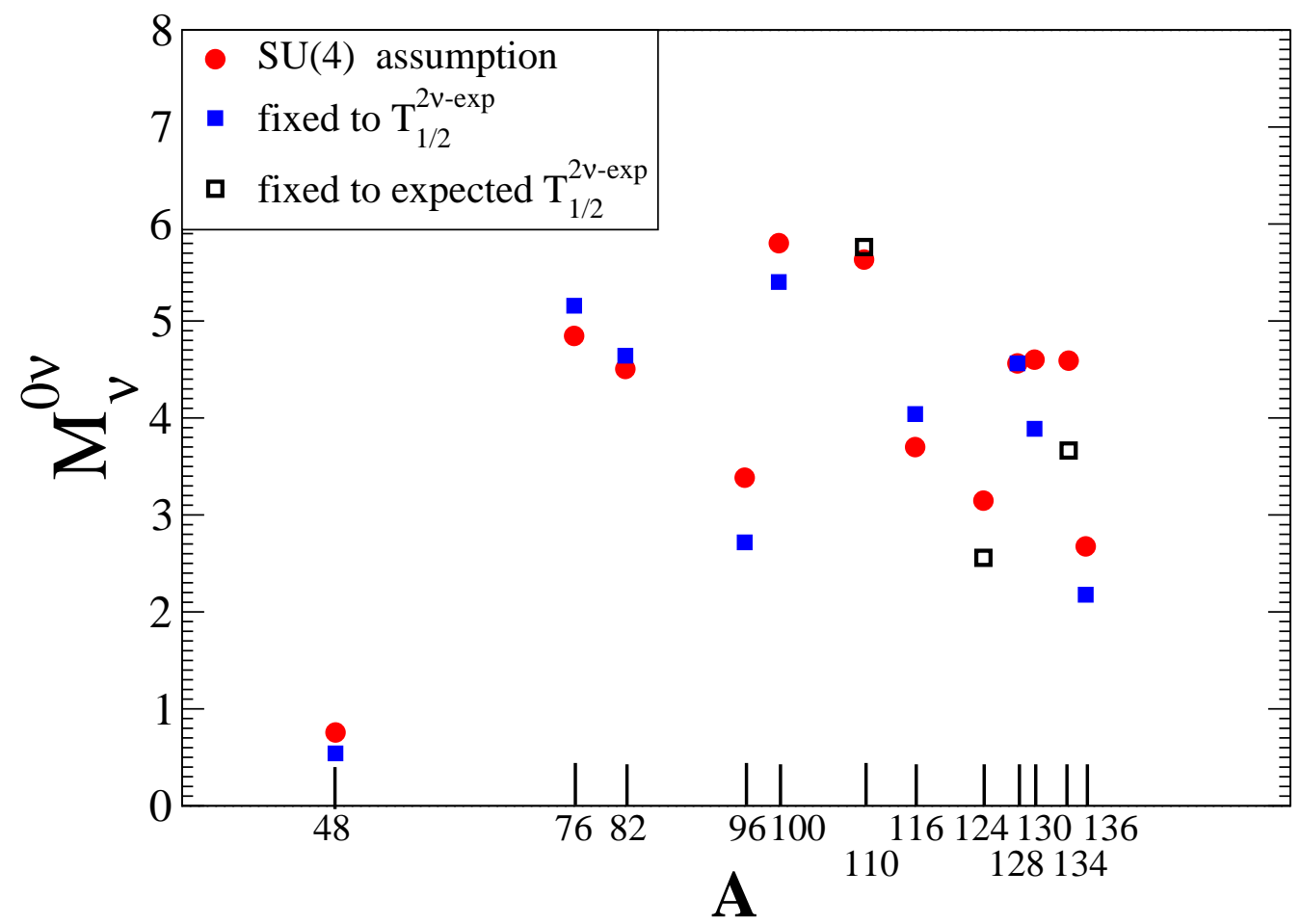

Figure 2: (Color online) Nuclear matrix elements $\left(M_{v}^{0 v}\right)$ for $0 v \beta \beta$-decay candidates as a function of mass number A. Results are from the QRPA [5]. The isoscalar particle-particle interaction is adjusted by a partial restoration of the the SU(4) symmetry (red circles) and that the $2 v \beta \beta$ decay experimental half-life is correctly reproduced (blue squares). In the case the $2 v \beta \beta$-decay half-life is not measured $\left({ }^{94} \mathrm{Zr},{ }^{110} \mathrm{Pd}\right.$, ${ }^{124} \mathrm{Sn}$, and ${ }^{134} \mathrm{Xe}$ ) the expected $2 v \beta \beta$-decay half-life, e.g., within the single-state dominance hypothesis, is considered (empty black squares). $g_{A}^{\text {eff }}=g_{A}^{\text {free }}=1.269$ is assumed.

Recently, the $0 v \beta \beta$-decay NMEs for nuclei of experimental interest were calculated by the Quasiparticle Random Phase Approximation (QRPA) by using a new way of adjusting the important parameter, namely the isoscalar particle-particle interaction [5]. A partial restoration of the spin-isospin $S U(4)$ symmetry, which corresponds to vanishing values of $M_{c l}^{2 v}$, was considered. The obtained values are not very different $(\leq 20 \%)$ from the usual QRPA values when isoscalar particleparticle interaction is related to the known $2 v \beta \beta$ half-lives. Fig. 2 compares NMEs produced by both ways of renormalization of this interaction. By assuming partial restoration of the SU(4) symmetry, the true $2 v \beta \beta$-decay matrix elements were evaluated and compared to the corresponding experimental values. It was found that calculated values are mostly larger than the experimental ones, suggesting qualitatively on average a relatively modest quenching $g_{A}^{\text {eff }}=0.71 g_{A}^{\text {free [5] }}$.

Recall that $g_{A}^{\text {eff }}$ enters in the fourth power in both the $0 v \beta \beta$ - and $2 v \beta \beta$-decay rates and is a major concern of experiments looking for a signal of the $0 v \beta \beta$-decay. There are some indications that its value can be significantly suppressed when compared to $g_{A}^{\text {free }}=1.269$ at nucleon level. 
By QRPA statistical analyses that took into account experimental EC and $\beta$ rates in addition to the measured $2 v \beta \beta$-decay half-lives, it was found $\left(g_{A}^{\text {eff }}\right)^{4}=0.30$ and 0.50 for the $2 v \beta \beta$-decay of ${ }^{100} \mathrm{Mo}$ and ${ }^{116} \mathrm{Cd}$, respectively [7]. A significant stronger quenching of the axial-vector coupling constant, namely $\left(g_{A}^{\text {eff }}\right)^{4} \simeq\left(1.269 A^{-0.18}\right)^{4}=0.063$, was established within the Interacting Boson Model by exploiting the $2 v \beta \beta$-decay matrix elements evaluated within closure approximation [8].

Experiments studying the $2 v \beta \beta$-decay are currently approaching a qualitatively new level, where high-precision measurements are performed not only for half-lives but also for differential characteristics of the process. Recently, by taking into account dependence of energy denominators on lepton energies via the Taylor expansion a more accurate expression for the $2 v \beta \beta$-decay halflife was derived [6]. The revised expression includes in addition to the well-known nuclear matrix element $M_{G T}^{2 v}$ also the double GT matrix element $M_{G T-3}^{2 v}$ with energy denominator to the third power. A novel method to determine $g_{A}^{\text {eff }}$ was proposed, which requires to measure a ratio of these two matrix elements from the energy and angular distributions of emitted electrons [6].

\section{Summary and Conclusion}

In summary, neutrino mass mechanisms of the $0 v \beta \beta$-decay associated with left and right leptonic currents were studied. An interpolating formula for the $0 v \beta \beta$-decay half-life, which allows to consider arbitrary neutrino mass, was introduced. It contains a single LNV parameter $\eta_{v N}$, which is a sum of squared parameters $m_{\beta \beta}$ and $M_{\beta \beta}^{R}$ characterizing left and right handed leptonic current mechanisms, respectively. It was manifested that an additional theoretical or experimental input about neutrino masses and mixing is needed to clarify the importance of each of these mechanisms. That goal was achieved by introducing $6 \times 6$ neutrino mixing matrix of seesaw type with minimal number of parameters.

A new development in the calculation of the $0 v \beta \beta$-decay NMEs within the QRPA was discussed. The role of the partial restoration of the spin-isospin SU(4) symmetry was indicated. The importance of the $2 v \beta \beta$-decay for a reliable evaluation of the $0 v \beta \beta$-decay NMEs was stressed and a relation between both types of NMEs was explained. The issue of possible significant quenching of $g_{A}$ was addressed. An approach to determine the value of $g_{A}^{\text {eff }}$ by exploiting $2 v \beta \beta$-decay differential characteristics was introduced.

\section{Acknowledgements}

This work is supported in part by the VEGA Grant Agency of the Slovak Republic under Contract No. 1/0922/16 and by the Ministry of Education, Youth and Sports of the Czech Republic under Contract No. LM2011027.

\section{References}

[1] J. Engel and J. Menéndez, Rept. Prog. Phys. 80, 046301 (2017).

[2] D. Štefánik, R. Dvornický, F. Šimkovic, and P. Vogel, Phys. Rev. C 92, 055502 (2015).

[3] F. Šimkovic, D. Štefánik, and R. Dvornický, Fron. in Phys. 5,57 (2017).

[4] A. Babič, S. Kovalenko, M.I. Krivoruchenko, and F. Šimkovic, Phys. Rev. D 98, 015003 (2018). 
[5] F. Šimkovic, A. Smetana, and P. Vogel, arXiv:1808.05016 [nucl-th], accepted in Phys. Rev. C

[6] F. Šimkovic, R. Dvornický, D. Štefánik, and A. Faessler, Phys. Rev. C 97, 034315 (2018).

[7] A. Faessler, G.L. Fogli, E. Lisi, V. Rodin, A.M. Rotunno, and F. Šimkovic, J. Phys. G 35, 075104 (2008).

[8] J. Barea, J. Kotila, and F. Iachello, Phys. Rev. C 87, 014315 (2013). 